\title{
Predictors for discrete time processes with energy decay on higher frequencies
}

\author{
Nikolai Dokuchaev \\ 1st version: December 7, 2011. Revised: May 22th, 2012
}

\begin{abstract}
The predictability of discrete-time processes is studied in a deterministic setting. A family of one-step-ahead predictors is suggested for processes of which the energy decays at higher frequencies. For such processes, the prediction error can be made arbitrarily small. The predictions can be robust with respect to the noise contamination at higher frequencies.

Index terms - Bandlimited, causal convolution, discrete time systems, harmonic analysis, prediction, Szegö-Kolmogorov Theorem.
\end{abstract}

MSC 2010 classification : 42A38, 93E10, 62M15, 42B30,

\section{Introduction}

The paper studies pathwise predictability of discrete time processes in a deterministic setting. It is well known that certain restrictions on the frequency distribution can ensure additional opportunities for prediction and interpolation of the processes. The classical result for continuous time processes is the Nyquist-Shannon-Kotelnikov interpolation theorem for the continuous time band-limited processes. These processes are presented in many models including econometrics models (see examples in [10]). In theory, it is not possible to conclude that a process is band-limited given some finite interval of observations. In practice, this conclusion is being made based on historical data for a certain process; this leads to models where processes are assumed to be band-limited. Predictability based on sampling and the Nyquist-Shannon-Kotelnikov theorem was discussed in [14], [5], [8],[13], [6] [7]. These references deal with the predictability of continuous-time, band-limited stochastic processes, which include stationary processes. The predictors obtained in this work were constructed for the setting where the shape of the spectral representation is supposed to be known. For discrete time processes, the predictability can also be achieved given some properties of spectral representations. For stationary discrete time Gaussian processes, Szegö-Kolmogorov Theorem ensures that the optimal prediction error is zero if the spectral

IEEE Transactions on Signal Processing, November 2012 60, No. 11, 6027-6030. Digital Object Identifier 10.1109/TSP.2012.2212436 
density $\phi$ is such that $\int_{-\pi}^{\pi} \log \phi\left(e^{i \omega}\right) d \omega=-\infty$ (see, e.g., [11], p.68). However, it was unknown how to construct a predictor when the shape of the spectral density is unknown. This long standing problem was addressed in [3]: a predictor for general type band-limited time series was suggested in a deterministic setting. This predictor was a modification of the predictor obtained in [1] for continuous time processes.

Unfortunately, there are serious limitations to the practical use of the predictors constructed for the band-limited processes. A common argument dismissing the effectiveness of these predictors is that the predictors are not robust with respect to small noise contamination. This leads to the conclusion that the predictability is an abnormality that disappears with the presence of arbitrarily small noise or some incompleteness of historical data.

This paper addresses these problems again. We consider discrete time processes with some restrictions on the rate of energy decay on the higher frequencies. We establish the predictability of these processes, and we suggest new linear predictors represented by causal convolution sums over past times representing historical observations (Theorem 1). The future values of the process are not supposed to be calculated precisely but are rather approximated with an error in a prescribed interval that can be made arbitrarily small uniformly over a wide class of underlying processes. Similarly to [3], the predictors are given explicitly in the frequency domain. Whereas the results of [3] were restricted to band-limited discrete-time processes, we now extend the analysis to processes that are not strictly band limited. The predictors suggested here are different from the predictors from [3]. The setting of the present paper is similar to the one for continuous time processes from [2], where predictors were suggested for processes that were not band-limited but were assumed to have an exponential rate of energy decay on higher frequencies.

These results sheds some new light on the predictability conundrum for band-limited processes. More precisely, it leads to the conclusion that the band-limited processes still allow robust predictability. The feasible predictability is an interval sense only, i.e., it produces an interval that contains the future value rather than the exact future value. The error can be made arbitrarily small; however, this would require a large enough norm value of the predictor's transfer function. We show that this prediction is robust with respect to noise contamination in the following sense. Given the size of the prediction error that is associated with a process that is free of noise contamination, the additional prediction error that is attributable to noise contamination depends linearly on the product of the noise with a norm associated with the transfer function entailed in forming the prediction from preceding values of the process (see Section IV). If the predictor is targeting too small a size of the error for processes without noise contamination, the norm of the transfer function increases, and this robustness vanishes.

The paper is organized in the following manner. In Section II, we formulate the definitions and the main result. In Section III, we prove the main theorem concerning the predictablility of processes with a certain rate of energy decay on higher frequencies. In Section IV, we discuss the robastness of the predictors with respect to noise contamination. Finally, in Section V, we summarize our results and offer suggestions for further research. 


\section{Definitions and main result}

Let $D \triangleq\{z \in \mathbf{C}:|z| \leq 1\}, D^{c}=\mathbf{C} \backslash D$, and $\mathbb{T} \triangleq\{z \in \mathbf{C}:|z|=1\}$.

We denote by $\ell_{r}$ the set of all sequences $x=\{x(t)\} \subset \mathbf{R}, t=0, \pm 1, \pm 2$, such that $\|x\|_{\ell_{r}}=$ $\left(\sum_{t=-\infty}^{\infty}|x(t)|^{r}\right)^{1 / r}<+\infty$ for $r \in[1, \infty)$ or $\|x\|_{\ell_{\infty}}=\sup _{t}|x(t)|<+\infty$ for $r=+\infty$.

Let $\ell_{r}^{+}$be the set of all sequences $x \in \ell_{r}$ such that $x(t)=0$ for $t=-1,-2,-3, \ldots$

For $x \in \ell_{1}$ or $x \in \ell_{2}$, we denote by $X=\mathcal{Z} x$ the Z-transform

$$
X(z)=\sum_{t=-\infty}^{\infty} x(t) z^{-t}, \quad z \in \mathbf{C} .
$$

Respectively, the inverse $x=\mathcal{Z}^{-1} X$ is defined as

$$
x(t)=\frac{1}{2 \pi} \int_{-\pi}^{\pi} X\left(e^{i \omega}\right) e^{i \omega t} d \omega, \quad t=0, \pm 1, \pm 2, \ldots
$$

If $x \in \ell_{2}$, then $\left.X\right|_{\mathbb{T}}$ is defined as an element of $L_{2}(\mathbb{T})$.

Let $H^{r}$ be the Hardy space of functions that are holomorphic on $D^{c}$ including the point at infinity (see, e.g., [4] ). Note that Z-transform defines a bijection between the sequences from $\ell_{2}^{+}$and the restrictions (i.e., traces) of the functions from $H^{2}$ on $\mathbb{T}$.

Definition 1 Let $\widehat{\mathcal{K}}$ be the class of functions $\widehat{k}: \ell_{\infty}^{+} \rightarrow \mathbf{R}$ such that $\widehat{k}(t)=0$ for $t<0$ and such that $\widehat{K}(\cdot)=\mathcal{Z} \widehat{k} \in H^{\infty}$.

Definition 2 Let $\mathcal{Y} \subset \ell_{r}$ be a class of processes.

(i) We say that this class is $\ell_{r}$-predictable if there exists a sequence $\left\{\widehat{k}_{m}(\cdot)\right\}_{m=1}^{+\infty} \subset \widehat{\mathcal{K}}$ such that

$$
\left\|x(t+1)-\widehat{x}_{m}(t)\right\|_{\ell_{r}} \rightarrow 0 \quad \text { as } \quad m \rightarrow+\infty \quad \forall x \in \mathcal{Y} .
$$

Here $\widehat{x}_{m}(t) \triangleq \sum_{s=-\infty}^{t} \widehat{k}_{m}(t-s) x(s)$.

(ii) We say that the class $\mathcal{Y}$ is uniformly $\ell_{r}$-predictable if, for any $\varepsilon>0$, there exists $\widehat{k}(\cdot) \in \widehat{\mathcal{K}}$ such that

$$
\|x(t+1)-\widehat{x}(t)\|_{\ell_{r}} \leq \varepsilon \quad \forall x \in \mathcal{Y} .
$$

Here $\widehat{x}(t) \triangleq \sum_{s=-\infty}^{t} \widehat{k}(t-s) x(s)$.

Let some $q>1$ be given. For $c>0$ and $\omega \in[-\pi, \pi]$, set

$$
h(\omega, c)=\exp \frac{c}{\left[(\cos (\omega)+1)^{2}+\sin ^{2}(\omega)\right]^{q / 2}} .
$$

Let $\mathcal{X}(c)$ be the class of all sequences $x \in \ell_{2}$ such that

$$
\underset{\omega \in[-\pi, \pi]}{\operatorname{ess} \sup }\left|X\left(e^{i \omega}\right)\right| h(\omega, c)<+\infty,
$$


where $X=\mathcal{Z} x$. Let $\mathcal{X}=\cup_{c>0} \mathcal{X}(c)$.

Note that $h(\omega, c) \rightarrow+\infty$ as $\omega \rightarrow \pm \pi$ and that (1) holds for degenerated processes, with $X\left(e^{i \omega}\right)$ approaching zero with sufficient rate of decay as $\omega \rightarrow \pm \pi$. In particular, the class $\mathcal{X}$ includes all bandlimited processes $x$ such that $X\left(e^{i \omega}\right)=0$ for $\omega \notin\left[-\omega_{1}, \omega_{1}\right]$, for some $\omega_{1} \in(0, \pi)$, where $X=\mathcal{Z} x$.

Theorem 1 Let either $r=2$ or $r=+\infty$.

(i) The class $\mathcal{X}$ is $\ell_{r}$-predictable.

(ii) Let $c_{0}>0$ be given, and let $\mathcal{U}\left(c_{0}\right)$ be a class of processes $x(\cdot) \in \mathcal{X}\left(c_{0}\right)$ such that

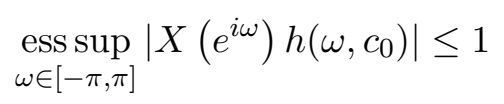

for $X=\mathcal{Z} x$. Then this class $\mathcal{U}\left(c_{0}\right)$ is uniformly $\ell_{r}$-predictable.

(iii) A sequence of predicting kernels that ensures prediction required in (i) and (ii) can be constructed as the following. Let $\mu>1$ be given. For $\gamma>0$, set

$$
\alpha=\alpha(\gamma)=1-\gamma^{\frac{2 \mu}{1-q}}, \quad V(z) \triangleq 1-\exp \left(-\frac{\gamma}{z+\alpha}\right), \quad \widehat{K}(z) \triangleq z V(z) .
$$

Then the required sequence of kernels that ensures prediction required in (i) and (ii) is $\widehat{k}(\cdot)=$ $\widehat{k}(\cdot, \gamma)=\mathcal{Z}^{-1} \widehat{K}$, where $\gamma=\gamma_{i} \rightarrow+\infty$. For these kernels,

$$
\|x(t+1)-\widehat{x}(t)\|_{\ell_{r}} \rightarrow 0 \quad \text { as } \quad \gamma \rightarrow+\infty \quad \forall x \in \mathcal{X} .
$$

Moreover, for any $c_{0}>0$ and $\varepsilon>0$, there exists $\gamma>0$ such that

$$
\|x(t+1)-\widehat{x}(t)\|_{\ell_{r}} \leq \varepsilon \quad \forall x \in \mathcal{U}\left(c_{0}\right) .
$$

Here $\widehat{x}(t) \triangleq \sum_{s=-\infty}^{t} \widehat{k}(t-s) x(s)$.

Note that any particular predictor described in Theorem 1 ensures predictability in an interval sense only, i.e., it produces an interval $[\widehat{x}(t+1)-\varepsilon, \widehat{x}(t+1)+\varepsilon]$ that contains $x(t+1)$ rather than the exact value of $x(t+1)$. However, this $\varepsilon$ can done arbitrarily via selection of a large enough $\gamma$.

The family of predicting kernels $\widehat{k}$ introduced above represents an extension on the discrete time setting of the construction introduced in [2] for continuous time processes with exponential rate of decay of energy on higher frequencies.

\section{Proofs}

In our setting, $x(t+1)$ is the output of anticausal convolution with the transfer function $K(z) \equiv z$, i.e., $x(t+1)=\mathcal{Z}^{-1}(K \mathcal{Z} x)(t)$.

Let $\Omega(\alpha)=\arccos (-\alpha)$, let $D_{+}(\alpha)=(-\Omega(\alpha), \Omega(\alpha))$, and let a $D(\alpha) \triangleq[-\pi, \pi] \backslash D_{+}(\alpha)$. We have that $\cos (\Omega(\alpha))+\alpha=0, \cos (\omega)+\alpha>0$ for $\omega \in D_{+}(\alpha)$, and $\cos (\omega)+\alpha<0$ for $\omega \in D(\alpha)$.

Note that $\alpha=\alpha(\gamma) \rightarrow 1$ as $\gamma \rightarrow+\infty$. 
Lemma 1 (i) $V(z) \in H^{\infty}$ and $\widehat{K}(z) \triangleq K(z) V(z) \in H^{\infty}$.

(ii) $V\left(e^{i \omega}\right) \rightarrow 1$ for all $\omega \in(-\pi, \pi)$ as $\gamma \rightarrow+\infty$.

(iii) If $\omega \in(-\Omega(\alpha), \Omega(\alpha))$ then $\operatorname{Re} W(\omega)>0$ and $\left|V\left(e^{i \omega}\right)-1\right| \leq 1$.

(iv) For any $c>0$, there exists $\gamma_{0}>0$ such that for any $\gamma \geq \gamma_{0}$ and for $V$ selected with $\alpha=\alpha(\gamma)$ we have $\int_{D(\alpha)}\left|V\left(e^{i \omega}\right)-1\right|^{\rho} h(\omega, c)^{-\rho} d \omega \leq 2 \arccos (\alpha)$ for any $\rho \geq 1$.

Proof of Lemma 1. Clearly, $V \in H^{\infty}$, and $z V(z)=K(z) V(z) \in H^{\infty}$, since the growth of $z$ is being compensated by multiplying with $V(p)=-\sum_{k=1}^{+\infty} \frac{1}{k !(z+\alpha)^{k}}$. Then statement (i) follows.

Further, for $\omega \in(-\pi, \pi)$, we have that

$$
\gamma \frac{1}{e^{i \omega}+\alpha}=\gamma \frac{e^{-i \omega}+\alpha}{\left|e^{i \omega}+\alpha\right|^{2}}
$$

Hence

$$
\operatorname{Re}\left(-\gamma \frac{1}{e^{i \omega}+\alpha}\right)=-\gamma \frac{\cos (\omega)+\alpha}{\left|e^{i \omega}+\alpha\right|^{2}}
$$

If $\gamma \rightarrow+\infty$ then $\alpha=\alpha(\gamma) \rightarrow 1$. This implies statements (ii)-(iii).

Let us prove statement (iv). We consider a large enough $\gamma$ such that $\alpha=\alpha(\gamma)>3 / 4$. For these $\alpha$ and $\omega \in D(\alpha)$, we have that $1 /\left|e^{i \omega}+\alpha\right|<2 /\left|e^{i \omega}+1\right|$. Hence $\gamma\left|\operatorname{Re}\left(1 /\left(e^{i \omega}+\alpha\right)\right)\right|<2 \gamma /\left|e^{i \omega}+1\right|$ for these $\alpha$ and $\omega$. Hence

$$
|V(i \omega)-1| h(\omega, c)^{-1} \leq \exp \left(\left|\operatorname{Re} \frac{\gamma}{e^{i \omega}+\alpha}\right|-\frac{c}{\left|e^{i \omega}+1\right|^{q}}\right) \leq \exp \left(\frac{2 \gamma}{\left|e^{i \omega}+1\right|}-\frac{c}{\left|e^{i \omega}+1\right|^{q}}\right)
$$

for all $\omega \in D(\alpha)$. Let $\rho(\alpha)=\left|e^{i \Omega(\alpha)}+1\right|^{-1}$. By the choice of $\alpha=\alpha(\gamma)$, it follows that

$$
\rho(\alpha)=(2-2 \alpha)^{-1 / 2}=\left(2 \gamma^{\frac{2 \mu}{1-q}}\right)^{-1 / 2}=2^{-1 / 2} \gamma^{\frac{\mu}{q-1}} .
$$

Hence

$$
2 \gamma \rho(\alpha)^{1-q}=2 \gamma\left(2^{-1 / 2} \gamma^{\frac{\mu}{q-1}}\right)^{1-q}=2^{1-(1-q) / 2} \gamma^{1-\mu}
$$

It follows that

$$
2 \gamma \rho(\alpha)^{1-q} \rightarrow 0 \quad \text { as } \quad \gamma \rightarrow+\infty
$$

By (3), for any $c>0$, there exists $\gamma_{0}>0$ such that $2 \gamma \rho(\alpha)^{1-q} \leq c$ for any $\gamma>\gamma_{0}$, and, therefore, $2 \gamma \rho(\alpha) \leq c \rho(\alpha)^{q}$. Moreover, $2 \gamma /\left|e^{i \omega}+1\right| \leq c /\left|e^{i \omega}+1\right|^{q}$ for all $\omega \in D(\alpha)$ since $1 /\left|e^{i \omega}+1\right| \geq \rho(\alpha)$ for these $\omega$. Hence

$$
|V(i \omega)-1| h(\omega, c)^{-1} \leq 1, \quad|V(i \omega)-1|^{\rho} h(\omega, c)^{-\rho} \leq 1
$$


for all $\omega \in D(\alpha)$. In addition, the measure of the set $D(\alpha)$ is $\pi-\arccos (-\alpha)+\arccos (\alpha)=$ $2 \arccos (\alpha)$. This completes the proof of statement (iv) and Lemma 1.

Proof of Theorem 1. Let $\gamma \rightarrow+\infty$, and let $V, K, \widehat{K}$ be as defined above. Let $k=\mathcal{Z}^{-1} K$ and $\widehat{k}=\mathcal{Z}^{-1} \widehat{K}$. For $x(\cdot) \in \mathcal{X}$, let $X \triangleq \mathcal{Z} x$ and

$$
y(t) \triangleq \sum_{s=t}^{\infty} k(t-s) x(s)=x(t+1), \quad \widehat{y}(t) \triangleq \sum_{s=-\infty}^{t} \widehat{k}(t-s) x(s) .
$$

We have that $\widehat{k}=\mathcal{Z}^{-1} \widehat{K}$ is real valued, since $k(\cdot)$ is real valued and $K(\bar{z})=\overline{K(z)}, K\left(e^{-i \omega}\right)=$ $\overline{K\left(e^{i \omega}\right)}$.

Let $Y\left(e^{i \omega}\right) \triangleq(\mathcal{Z} y)\left(e^{i \omega}\right)=K\left(e^{i \omega}\right) X\left(e^{i \omega}\right)$. By the definitions, it follows that $\widehat{Y}\left(e^{i \omega}\right) \triangleq$ $\widehat{K}\left(e^{i \omega}\right) X\left(e^{i \omega}\right)=Y(\mathcal{Z} \widehat{y})\left(e^{i \omega}\right)$.

Further, let $\rho=2$ if $r=2$ and $\rho=1$ if $r=+\infty$.

We have that $\left\|\widehat{Y}\left(e^{i \omega}\right)-Y\left(e^{i \omega}\right)\right\|_{L_{\rho}(-\pi, \pi)}^{\rho}=I_{1}+I_{2}$, where

$$
I_{1}=\int_{D(\alpha)}\left|\widehat{Y}\left(e^{i \omega}\right)-Y\left(e^{i \omega}\right)\right|^{\rho} d \omega, \quad I_{2}=\int_{D_{+}(\alpha)}\left|\widehat{Y}\left(e^{i \omega}\right)-Y\left(e^{i \omega}\right)\right|^{\rho} d \omega .
$$

By the assumptions, there exists $c>0$ such that $\left\|X\left(e^{i \omega}\right) h(\omega, c)\right\|_{L_{\infty}(-\pi, \pi)}<+\infty$. Hence

$$
\begin{aligned}
I_{1}^{1 / \rho} & =\left\|\widehat{Y}\left(e^{i \omega}\right)-Y\left(e^{i \omega}\right)\right\|_{L_{\rho}(D(\alpha))}=\left\|\left(\widehat{K}\left(e^{i \omega}\right)-K\left(e^{i \omega}\right)\right) X\right\|_{L_{\rho}(D(\alpha))} \\
& \leq\left\|\left(V\left(e^{i \omega}\right)-1\right) h(\omega, c)^{-1}\right\|_{L_{\rho}(D(\alpha))}\left\|K\left(e^{i \omega}\right) X\left(e^{i \omega}\right) h(\omega, c)\right\|_{L_{\infty}(-\pi, \pi)} \\
& \leq(2 \arccos (\alpha))^{1 / \rho}\left\|X\left(e^{i \omega}\right) h(\omega, c)\right\|_{L_{\infty}(-\pi, \pi)} .
\end{aligned}
$$

The last inequality holds by Lemma 1 (iv). It follows that $I_{1} \rightarrow 0$ as $\gamma \rightarrow+\infty$.

Let us estimate $I_{2}$. Lemma 1 (iii) gives that $\left|V\left(e^{i \omega}\right)-1\right| \leq 1$ for all $\omega \in D_{+}(\alpha)$. We have that

$$
\begin{array}{r}
I_{2}=\int_{D_{+}(\alpha)}\left|K\left(e^{i \omega}\right)\left(1-V\left(e^{i \omega}\right)\right) X\left(e^{i \omega}\right)\right|^{\rho} d \omega \leq \psi(\gamma)\left\|X\left(e^{i \omega}\right)\right\|_{L_{\infty}(-\pi, \pi)} \\
\leq \psi(\gamma)\left\|X\left(e^{i \omega}\right) h(\omega, c)\right\|_{L_{\infty}(-\pi, \pi)}^{\rho},
\end{array}
$$

where

$$
\psi(\gamma)=\int_{D_{+}(\alpha)}\left|K\left(e^{i \omega}\right)\left(1-V\left(e^{i \omega}\right)\right)\right|^{\rho} d \omega=\int_{-\pi}^{\pi} \mathbb{I}_{D_{+}(\alpha)}(\omega)\left|K\left(e^{i \omega}\right)\left(1-V\left(e^{i \omega}\right)\right)\right|^{\rho} d \omega .
$$

Here $\mathbb{I}$ denotes the indicator function.

By Lemma 1(ii), $\mathbb{I}_{D_{+}(\alpha)}(\omega)\left|K\left(e^{i \omega}\right)\left(1-V\left(e^{i \omega}\right)\right)\right|^{\rho} \rightarrow 0$ a.e. as $\gamma \rightarrow+\infty$. By Lemma 1(iii),

$$
\mathbb{I}_{D_{+}(\alpha)}(\omega)\left|K\left(e^{i \omega}\right)\left(1-V\left(e^{i \omega}\right)\right)\right|^{\rho} \leq \sup _{\omega \in D_{+}(\alpha)}\left|K\left(e^{i \omega}\right)\right|^{\rho} \leq 1 .
$$

From Lebesgue Dominance Theorem, it follows that $\psi(\gamma) \rightarrow 0$ as $\gamma \rightarrow+\infty$. It follows that $I_{1}+I_{2} \rightarrow 0$ for any $c>0, x \in \mathcal{X}(c)$. By the definition of $\rho$, we have that $1 / \rho+1 / r=1$. Hence $\|\widehat{y}-y\|_{\ell_{r}} \rightarrow 0$ as $\gamma \rightarrow+\infty$ for any $x \in \mathcal{X}$. This completes the proof of statement (i). 
Let us prove statement (ii). We have that

$$
\begin{array}{r}
\left\|\widehat{Y}\left(e^{i \omega}\right)-Y\left(e^{i \omega}\right)\right\|_{L_{\rho}(-\pi, \pi)}^{\rho}=I_{1}+I_{2} \leq(2 \arccos (\alpha)+\psi(\gamma))\left\|X\left(e^{i \omega}\right) h\left(\omega, c_{0}\right)\right\|_{L_{\infty}(-\pi, \pi)}^{\rho} \\
\leq 2 \arccos (\alpha)+\psi(\gamma)
\end{array}
$$

for any $x \in \mathcal{U}\left(c_{0}\right)$. For any $\varepsilon>0$, one can select $\gamma$ such that $\psi(\gamma) \leq \varepsilon^{\rho} / 2$ and that $2 \arccos (\alpha) \leq \varepsilon^{\rho} / 2$. This choice ensures that $\|\widehat{y}-y\|_{\ell_{r}} \leq \varepsilon$. This completes the proof of statement (ii). It follows that the predicting kernels $\widehat{k}(\cdot)=\mathcal{Z}^{-1} \widehat{K}$ are such as required. This completes the proof of Theorem 1 .

It can be noted that the choice of predicting kernels is not unique. In particular, the kernels preserve the properties described in Theorem 1 for any selection of $\alpha=\alpha(\gamma)$ such that (3) holds. For instance, $\alpha(\gamma)$ can be selected as

$$
\alpha=\alpha(\gamma)=1-(\log \gamma)^{-1} \gamma^{\frac{2}{1-q}}
$$

In addition, it follows from the proofs that the uniform predictability from statement (ii) of Theorem 1 can be ensured with

$$
\alpha=\alpha(\gamma)=1-\frac{1}{2}\left(2 \gamma / c_{0}\right)^{\frac{2}{1-q}} .
$$

In this case, $\rho(\alpha)=\left(2 \gamma / c_{0}\right)^{1 /(q-1)}$. This corresponds to the case where $\mu=1$ in Theorem 1(iii).

\section{On the prediction error generated by noise contamination}

Let us estimate the prediction error for the case when the predictor designed for processes from $\mathcal{X}$ is applied to a process with a small high-frequency noise contamination. Let us consider a process $x(\cdot) \in$ $\ell_{\infty}$ such that $x=x_{0}+x_{N}$, where $x_{0} \in \mathcal{X}, x_{N} \in \ell_{\infty}$. The process $x_{N}$ represents the noise. Let $X=\mathcal{Z} x$, $X_{0}=\mathcal{Z} x_{0}$, and $X_{N}=\mathcal{Z} x_{N}$. We assume that $X_{0}\left(e^{i \omega}\right) \in L_{1}(-\pi, \pi)$ and $\left\|X_{N}\left(e^{i \omega}\right)\right\|_{L_{1}(-\pi, \pi)}=\nu$. The parameter $\nu \geq 0$ represents the intensity of the noise.

Assume that the predictor is constructed as in Theorem 1 under the hypothesis that $\nu=0$ (i.e, that $x_{N}=0$ and $x \in \mathcal{X}$ ). For an arbitrarily small $\varepsilon>0$, there exists $\gamma$ such that, if the hypothesis that $\nu=0$ is correct, then

$$
\int_{-\pi}^{\pi}\left|\left(\widehat{K}\left(e^{i \omega}\right)-K\left(e^{i \omega}\right)\right) X\left(e^{i \omega}\right)\right| d \omega=\int_{-\pi}^{\pi}\left|\left(\widehat{K}\left(e^{i \omega}\right)-K\left(e^{i \omega}\right)\right) X_{0}\left(e^{i \omega}\right)\right| d \omega \leq 2 \pi \varepsilon
$$

and

$$
\|\widehat{y}-y\|_{\ell_{\infty}} \leq \varepsilon
$$

Let us estimate the prediction error for the case where $\nu>0$. We have that

$$
\|\widehat{y}-y\|_{\ell_{\infty}} \leq J_{0}+J_{N}
$$


where

$J_{0}=\frac{1}{2 \pi}\left\|\left(\widehat{K}\left(e^{i \omega}\right)-K\left(e^{i \omega}\right)\right) X_{0}\left(e^{i \omega}\right)\left|\left\|_{L_{1}(-\pi, \pi)}, \quad J_{N}=\frac{1}{2 \pi}\right\|\left(\widehat{K}\left(e^{i \omega}\right)-K\left(e^{i \omega}\right)\right) X_{N}\left(e^{i \omega}\right)\right|\right\|_{L_{1}(-\pi, \pi)}$.

The value $J_{N}$ represents the additional error caused by the presence of unexpected high-frequency noise (when $\nu>0$ ). It follows that

$$
\|\widehat{y}-y\|_{\ell_{\infty}} \leq \varepsilon+\nu(\kappa+1)
$$

where $\kappa=\sup _{\omega \in[-\pi, \pi]}\left|\widehat{K}\left(e^{i \omega}\right)\right|$.

Therefore, it can be concluded that the prediction is robust with respect to noise contamination for any given $\varepsilon$. On the other hand, if $\varepsilon \rightarrow 0$ then $\gamma \rightarrow+\infty$ and $\kappa \rightarrow+\infty$. In this case, error (4) is increasing for any given $\nu>0$. This happens when the predictor is targeting too small a size of the error for the processes from $\mathcal{X}$, i.e., under the assumption that $\nu=0$.

The equations describing the dependence of $\varepsilon$ and $\kappa$ on $\gamma$ could be derived similarly to estimates in [3], Section 6, where it was done for different predicting kernels and for band-limited processes. We leave it for future research.

\section{Concluding remarks}

(i) Technically, the predictors obtained above require the past values of $x(s)$ for all $s \in(-\infty, t]$. However, $\sum_{s=-\infty}^{t} \widehat{k}(t-s) x(s)$ can be approximated by $\sum_{s=-M}^{t} \widehat{k}(t-s) x(s)$ for a large enough $M>0$. Therefore, the predictors are robust with respect to replacing the semi-infinite time interval of observations by a large finite one.

(ii) For processes from $\mathcal{X}$, the selection of a large enough $\gamma$ can ensure that the prediction error is arbitrarily small. However, the corresponding predictor transfer function $\widehat{K}$ is large in norm for large $\gamma$. This leads to a large error caused by any noise contamination presented for processes $x(\cdot) \notin \mathcal{X}$, i.e., with the energy on higher frequencies that is not decaying fast enough near the point $z=e^{i \pi}$. Nevertheless, the suggested predictors are robust with respect to the contamination noise for any fixed $\gamma$. The error generated by the noise is limited if $\kappa$ in (4) is limited, i.e., if $\gamma$ is limited and $\varepsilon$ in (4) is not too small.

(iii) The presence of robustness mentioned above leads to the conclusion that certain interval type predictability for band-limited processes is not an abnormality that disappears with the presence of arbitrarily small noise or some incompleteness of historical data. This predictability holds even for processes with a certain rate of energy decay on higher frequencies that are not exactly band-limited. This must be taken into account for all models where band-limited processes are assumed. In particular, this implies that band-limited processes should be used with caution in the models where predictability is difficult to justify such as models for financial time series. 
(iv) The results of this paper can be applied to discrete time stationary random Gaussian processes with the spectral densities $\phi$ such that $\int_{-\pi}^{\pi} \phi\left(e^{i \omega}\right) h(\omega, c) d \omega<+\infty$, i.e., when the spectral density is decaying fast enough on the higher frequencies. By Szegö-Kolmogorov Theorem, it was known in principle that the minimal (optimal) predicting error is zero in this case. However, it was unknown how to construct the corresponding predictors for general classes of $\phi$.

(v) The restrictions on the spectral representations imposed on the underlaying processes are quite tight. They are not satisfied for the samples generated from autoregressive stochastic models including ARMA, FARIMA, FEXP and other common long-memory processes (see, e.g., [12]). It is yet unclear in which applications the processes with the required rate of energy decay on higher frequencies could be found. Therefore, it is unclear where to find real data sets to test the suggested predictors. We may suggest applying the corresponding predictors for band-limited processes such as described in [10]. We leave it for future research.

\section{Acknowledgment}

This work was supported by ARC grant of Australia DP120100928 to the author. The author thanks Prof. Augusto Ferrante for useful discussion and advice on the time series analysis.

\section{References}

[1] N.Dokuchaev, "The predictability of band-limited, high-frequency, and mixed processes in the presence of ideal low-pass filters," Journal of Physics A: Mathematical and Theoretical 41, No 38, 382002, 2008. (7pp).

[2] N. Dokuchaev, "Predictability on finite horizon for processes with exponential decrease of energy on higher frequencies", Signal Processing 90, Iss. 2, 2010, pp. 696-701.

[3] N.Dokuchaev, "On predictors for band-limited and high-frequency time series", Signal Processing 92, iss. 10, 2010, pp. 2571-2575.

[4] P. Duren, Theory of $H^{p}$-Spaces. Academic Press, New York, 1970.

[5] J.J. Knab, "Interpolation of band-limited functions using the approximate prolate series", IEEE Transactions on Information Theory 25 (6), 1979, pp. 717-720.

[6] R.J. Lyman, W.W. Edmonson, S. McCullough, and M. Rao, "The predictability of continuoustime, bandlimited processes," IEEE Transactions on Signal Processing 48, Iss. 2, 2000, pp. 311316.

[7] R.J. Lyman and W.W. Edmonson, "Linear prediction of bandlimited processes with flat spectral densities.," IEEE Transactions on Signal Processing 49, Iss. 7, 2001, pp. 1564-1569. 
[8] F. Marvasti, "Comments on "A note on the predictability of band-limited processes,"” Proceedings of the IEEE 74 (11), 1986, p. 1596.

[9] A. Papoulis, "A note on the predictability of band-limited processes," Proceedings of the IEEE 73 (8), 1985, pp. 1332-1333.

[10] D.S.G. Pollock, "Band-Limited Stochastic Processes in Discrete and Continuous Time," Studies in Nonlinear Dynamics \& Econometrics 16, Iss. 1, Paper 1, 2012, pp. 1-28.

[11] M. Pourahmadi, "Foundations of time series analysis and prediction theory". Wiley, NY, 2001.

[12] P.M. Robinson, Time Series with Long Memory. Oxford University Presss, New York, 2003.

[13] P.P. Vaidyanathan, "On predicting a band-limited signal based on past sample values," Proceedings of the IEEE 75 (8), 1987, pp. 1125-1127.

[14] L.A. Wainstein and V.D. Zubakov, Extraction of Signals from Noise. Englewood Cliffs, NJ: Prentice-Hall, 1962. 